\title{
High-Frequency Oscillations Are Not Necessary for Simple Olfactory Discriminations in Young Rats
}

\author{
Max L. Fletcher, Abigail M. Smith, Aaron R. Best, and Donald A. Wilson \\ Department of Zoology, University of Oklahoma, Norman, Oklahoma 73072
}

Individual olfactory bulb mitral/tufted cells respond preferentially to groups of molecularly similar odorants. Bulbar interneurons such as periglomerular and granule cells are thought to influence mitral/tufted odorant receptive fields through mechanisms such as lateral inhibition. The mitral-granule cell circuit is also important in the generation of the odor-evoked fast oscillations seen in the olfactory bulb local field potentials and hypothesized to be an important indicator of odor quality coding. Infant rats, however, lack a majority of these inhibitory interneurons until the second week of life. It is unclear if these developmental differences affect olfactory bulb odor coding or behavioral odor discrimination. The following experiments are aimed at better understanding odor coding and behavioral odor discrimination in the developing olfactory system. Single-unit recordings from mitral/tufted cells and local field-potential recordings from both the olfactory bulb and anterior piriform cortex were performed in freely breathing urethane-anesthetized rats (postnatal day 7 to adult). Age-dependent behavioral odor discrimination to a homologous series of ethyl esters was also examined using a crosshabituation paradigm. Odorants were equated in all experiments for concentration $(150 \mathrm{ppm})$ using a flow dilution olfactometer. In concordance with the reduced interneuron population, local field potentials in neonates lacked detectable odor-evoked $\gamma$-frequency oscillations that were observed in mature animals. However, mitral/tufted cell odorant receptive fields and behavioral odor discrimination did not significantly change, despite known substantial changes in local circuitry and neuronal populations, over the age range examined. The results suggest that high-frequency local field-potential oscillations do not reflect processes critical for simple odor discrimination.

Key words: olfactory bulb; piriform cortex; receptive fields; cross-habituation; memory; local field-potential oscillations

\section{Introduction}

The olfactory bulb is thought to function as a combinatorial odorant feature detector in which different parts of odor molecules activate specific subsets of bulbar output neurons. This code is then sent to higher cortical centers involved in integrating this information into perceptual wholes (odor objects) involved in sensory discrimination and memory (Wilson and Stevenson, 2003).

Two views of olfactory bulb combinatorial coding exist. First, glomeruli and their corresponding mitral/tufted cells respond preferentially to groups of molecularly similar odorants (Uchida et al., 2000; Leon and Johnson, 2003), resulting in odor-specific spatial patterns of glomerular activity and associated mitral/ tufted cell output. Through mechanisms such as glomerular and granule cell layer lateral inhibition, inhibitory interneurons could shape the spatiotemporal activity pattern to enhance contrast between similar odorant features, as well as begin the process of feature synthesis.

\footnotetext{
Received Nov. 15, 2004; revised Dec. 2, 2004; accepted Dec. 4, 2004.

This work was supported by grants from the National Institute on Deafness and 0ther Communication Disorders (NIDCD) and the Oklahoma Center for the Advancement of Science and Technology to D.A.W. and an NIDCD predoctoral fellowship to M.L.F. We thank Brent Meyers for technical assistance.

Correspondence should be addressed to Donald A. Wilson, Department of Zoology, University of Oklahoma, Norman, 0K 73019. E-mail: dwilson@ou.edu.

DOI:10.1523/JNEUROSCI.4673-04.2005

Copyright $\odot 2005$ Society for Neuroscience $\quad$ 0270-6474/05/250792-07\$15.00/0
}

A second proposed coding mechanism involves coincident firing of groups of mitral/tufted cells precisely synchronized to oscillatory local field potentials (LFPs). Oscillatory activity has been found in the olfactory systems of many species (Adrian, 1950; Bressler and Freeman, 1980; Laurent and Davidowitz, 1994; Lam et al., 2000). In some invertebrates, odor discrimination is thought to rely on the precise oscillatory synchronization of dynamic olfactory bulb assemblies in which coincident firing of projection cells would form the basis of feature synthesis and odor coding (Laurent, 2002), although other studies have shown that projection cell synchronization is not dependent on these oscillations (Christensen et al., 2003). In this model, the role of inhibitory interneurons in the shaping of individual projection cell tuning curves is less important than their role in precisely synchronizing subsets of active cells to the global oscillatory dynamics of the bulb (Laurent, 1999).

Odors elicit both $\beta$-frequency (15-40 Hz) and $\gamma$-frequency (40-75 Hz) oscillations in the mammalian olfactory bulb, although their strict relationship to odor quality coding is unclear (Adrian, 1950; Bressler and Freeman, 1980; Chabaud et al., 2000; Neville and Haberly, 2003). Oscillations in mammalian olfactory bulb may reflect odor quality coding (Nusser et al., 2001), but have also been linked to memory (Ravel et al., 2003) and experience-dependent phenomena such as expectancy (Kay et al., 1996). In this latter view, inhibitory neurons such as granule cells serve as the target of cortical feedback circuits to modify bulb 
activity based on changes in expectation and state (Freeman, 1978). In this model, therefore, disruption of normal inhibitory interneuron function may not be expected to produce drastic effects on simple odor discrimination.

Infant rats offer an excellent system for investigating olfactory bulb odor coding mechanisms in that they possess a morphologically and physiologically immature olfactory system and yet are capable of, and in fact are dependent on, olfactory learning and odor-guided behaviors (Wilson and Sullivan, 1994). For example, neonatal rats lack a majority of bulbar inhibitory interneurons thought to be involved in generating fast oscillations (Rosselli-Austin and Altman, 1979; Mair et al., 1982), and thus lack adult-like spontaneous local oscillatory activity (Almli et al., 1985). However, it is unclear if these developmental differences affect olfactory bulb coding necessary for simple behavioral odor discrimination. The following experiments were aimed at better understanding olfactory bulb oscillatory activity, mitral/tufted cell olfactory receptive fields (ORFs), and how they relate to odor coding and odor discrimination in the developing olfactory system.

\section{Materials and Methods}

Subjects. Male and female Long-Evans hooded rats (Harlan, Indianapolis, IN) were used. Rats were housed in polypropylene cages with food and water available ad libitum. Lights were maintained on a $12 \mathrm{~h}$ light/ dark cycle with all testing during the light hour cycle. Animal care and protocols were approved by the University of Oklahoma Institutional Animal Care and Use Committee in accordance with National Institutes of Health guidelines. Postnatal day 7 (P7) (mean weight, $16.6 \pm 0.3 \mathrm{~g}$ ), P14 (mean weight, $32.3 \pm 0.8 \mathrm{~g}$ ), and mature rats (mean weight, $264.4 \pm$ $31.3 \mathrm{~g}$ ) were compared. Day of birth was considered P0. P7 rats were chosen because they have previously been shown to have dramatically reduced numbers of granule cells and reduced numbers of juxtaglomerular neurons, although relatively normal numbers of mitral cells (Brunjes and Frazier, 1986). Many mitral cells at this age, however, have multiple apical dendrites extending into more than one glomerulus (Malun and Brunjes, 1996). Similar results have been described in mice, with the addition that some classes of olfactory receptor neuron axons extend to more than one glomerulus at this age, before being pruned back in older mice (Zou et al., 2004).

Olfactory bulb physiology. For LFP and single-unit mitral/tufted cell recordings, animals were anesthetized with urethane $(1.5 \mathrm{~g} / \mathrm{kg})$ and placed in a stereotaxic apparatus. After exposure of the skull, a small hole was drilled over the left olfactory bulb for bulbar recordings or over the left anterior piriform cortex for cortical recordings. LFP and single-unit recordings at all ages were aimed at a similar dorsomedial region of the olfactory bulb. Another small hole was drilled posterior to the bulb, allowing a tungsten stimulating electrode to be placed in the ipsilateral lateral olfactory tract for identification of mitral/tufted cells through antidromic activation. A piezoelectric monitor strapped around the animal's chest monitored respiratory activity throughout the experiment. The output was then sent to a window discriminator allowing the odorant pulse to be delivered on the transition of the inspiration-expiration cycle. Animals were placed on a heating pad to maintain constant body temperature.

Field-potential recordings were made with tungsten microelectrodes (bulb) ( $5 \mathrm{M} \Omega$; A-M Systems, Carlsburg, WA) or stainless steel wire (cortex) $(0.3 \mathrm{~mm}$, Teflon-coated; A-M Systems). All penetrations were approximately perpendicular to the brain surface. Signals were amplified $1000 \times$, bandpass-filtered $(0.1-10,000 \mathrm{~Hz})$, acquired at $500 \mathrm{~Hz}$, and analyzed using Spike 2 software (Cambridge Electronic Design, Cambridge, UK). Spectral analysis was performed on $3 \mathrm{~s}$ windows of data immediately before and after odorant onset using fast Fourier transforms after application of a Hanning window. Odor-evoked power spectra were normalized to the baseline preodor power for each frequency to facilitate comparisons of relative odor-evoked power increases for each frequency band for each age.
A small number of animals were also used to confirm that differences in local field-potential activity across age did not simply reflect differences in anesthetic state. Animals (P7) were anesthetized with pentobarbital and had chronic indwelling stainless steel electrodes implanted into the olfactory bulb, and were allowed to recover (Wilson and Sullivan, 1990). Odor-evoked local field-potential activity was analyzed as described for anesthetized animals.

Single-unit recordings were made with tungsten microelectrodes (5 $\mathrm{M} \Omega$; A-M Systems). Signals were amplified, bandpass-filtered $(300 \mathrm{~Hz}$ to $10 \mathrm{kHz}$ ), acquired at $25 \mathrm{kHz}$, and analyzed using Spike 2 software (Cambridge Electronic Design). Responses to a homologous series of ethyl esters were compared in a total of 77 cells from P7 and mature rats. Only cells showing excitatory responses to at least one of the ethyl esters presented were used in this study. For all RF mapping, animals were given $2 \mathrm{~s}$ odor pulses delivered in pseudorandom order. Response magnitudes were calculated by subtracting the number of spikes during a 4 s prestimulus period from the number of spikes during each $4 \mathrm{~s}$ odorant presentation. In most animals, odorants were presented at least twice, and the mean response for each odorant was used. To allow for comparisons of overall RF shape, normalized RFs were obtained by calculating response magnitudes for each odorant as a percentage of the bestodorant response magnitude as previously described (Fletcher and Wilson, 2003).

Mean single-unit spontaneous activity was calculated from the spontaneous firing rate of each cell for each $4 \mathrm{~s}$ period immediately preceding all odor presentations. Odor-evoked mitral/tufted cell mean maximum instantaneous firing frequency was calculated from the maximum firing rate of each cell for each $4 \mathrm{~s}$ period immediately after odor onset. Analysis for relating spike activity to respiratory cycle was calculated by constructing phase histograms of unit activity relative to the respiratory cycle for

\section{A}
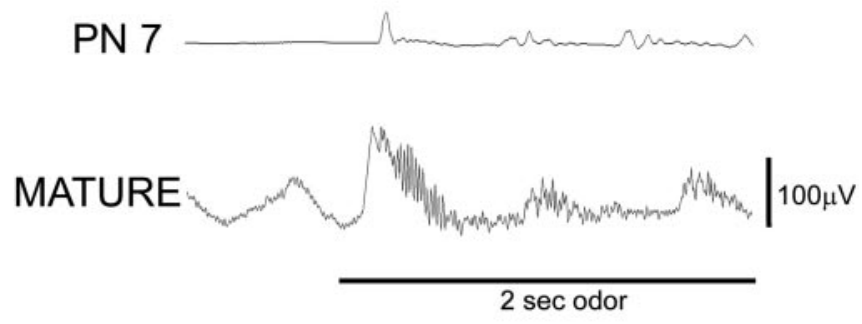

B
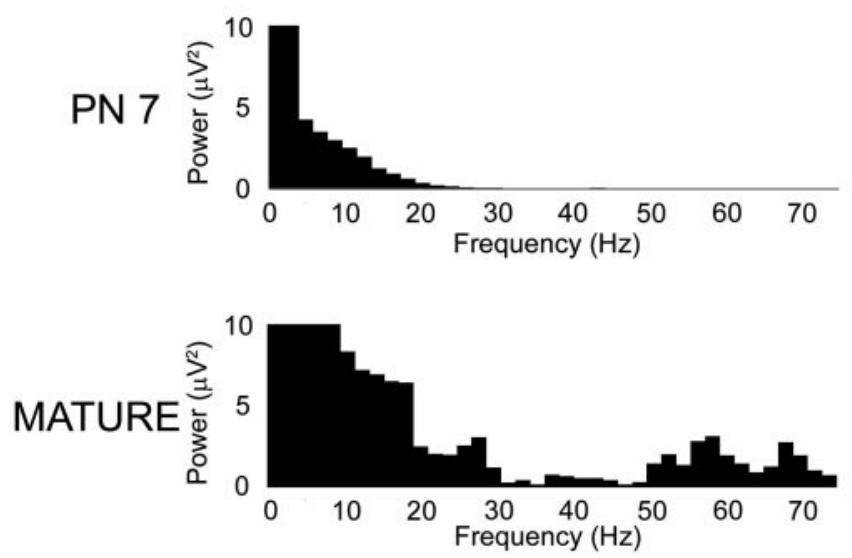

Figure 1. Examples of olfactory bulb LFPs recorded before and during a $2 \mathrm{~s}$ presentation of isoamyl acetate (150 ppm dilution). A, Raw signal recorded from the olfactory bulb of a 7-d-old and a mature rat, respectively. The horizontal bar indicates odor presentation. $B$, Power spectra for the odor-evoked oscillations taken from LFP examples above. Note that the majority of odor-evoked activity in the $\mathrm{P} 7$ rat is in the lower frequency ranges with a minimal $\beta$ band $(15-40 \mathrm{~Hz})$ response and no $\gamma$ band $(40-75 \mathrm{~Hz})$ activity compared with the mature rat analysis showing a relatively large increase in both $\beta$ and $\gamma$ band odor-evoked activity. 
$4 \mathrm{~s}$ after each odor onset. Mean vectors describing unit activity and respiratory cycle phase relationships were determined and compared across ages, as described in Wilson (1998), with a vector length of 0 representing no phase relationship between unit activity and respiration and a vector length of 1 representing phase lock between respiration and unit activity.

Odor discrimination. Postnatal day $7(n=7)$, postnatal day $14(n=11)$, or mature rats were used $(n=9)$. For P14 and mature animals, telemetry devices were used to measure heart rate (Data Sciences International, St. Paul, MN). The devices were implanted subcutaneously on the dorsal surface under sodium pentobarbital anesthesia $(50 \mathrm{mg} / \mathrm{kg})$. After recovery from the anesthetic, the P14 pups were placed back with their mothers for $2 \mathrm{~d}$. For P7 rats, subcutaneous electrodes were implanted under isoflurane anesthesia as described previously (Fletcher and Wilson, 2001). After recovery pups were placed in the testing chamber and allowed a $15 \mathrm{~min}$ recovery period before testing. Animals were given $4 \mathrm{~s}$ presentations of the odorants. Only animals displaying heart rate drops to at least two odors were used. In these animals, one odor was chosen as the habituating odor. Habituation consisted of 20,4 s odor presentations given every $10 \mathrm{~s}$. After habituation, the other odors were presented again. The final habituation and cross-habituation magnitudes were calculated as a percentage of the initial heart rate drop for that odor.

Odorant stimulation. For all experiments, odorant concentrations were $150 \mathrm{ppm}$. Odorants were delivered by passing a stream of humidified, charcoal-filtered air through vials containing each odorant using a flow dilution olfactometer. Odorants used were ethyl acetate, ethyl propionate, ethyl butyrate, ethyl valerate, ethyl hexanoate, ethyl heptanoate, ethyl octanoate, methyl butyrate, propyl butyrate, and isoamyl acetate (Sigma, St. Louis, MO).

\section{Results}

Odor stimulation evoked quite different oscillatory activity in the olfactory bulbs of 7-d-old compared with mature rats. As can be seen in Figure $1 A$, the majority of odor-evoked activity seen in the olfactory bulb of P7 rats consisted of the same low-frequency oscillations seen before odor onset as well as a minimal $\beta$ band $(15-40 \mathrm{~Hz})$ response and essentially no detectable $\gamma$ band $(40-75$ $\mathrm{Hz}$ ) activity (Fig. $1 B$ ). The odor-evoked LFP recorded in the mature olfactory bulb showed much greater oscillatory bursts per respiratory cycle (Fig. 1A). These oscillatory bursts contained greater $\beta$ band $(15-40 \mathrm{~Hz})$ and $\gamma$ band $(40-75 \mathrm{~Hz})$ activity in the mature olfactory bulb (Fig. $1 B$ ).

Mean raw power spectra (Fig. $2 A$ ) for the odor-evoked oscillations demonstrate that the majority of odor-evoked activity in the P7 rat is in the lower frequency ranges. This consisted of a minimal $\beta$ band response and very little $\gamma$ band activity compared with the mature rats in which there was a relatively large increase in both $\beta$ and $\gamma$ band odor-evoked activity. Mean raw power spectra were normalized to account for differences in overall baseline power across age. The normalized data again showed the overall trend of increases in both odor-evoked $\beta$ band and $\gamma$ band power in mature rats, whereas the $\mathrm{P} 7$ rats had a slight increase in odor-evoked $\beta$ band activity with a very large relative increase in lower-frequency oscillations (Fig. $2 B$ ).

Time-frequency comparisons of total $\beta$ and $\gamma$ band activity by
PN 7
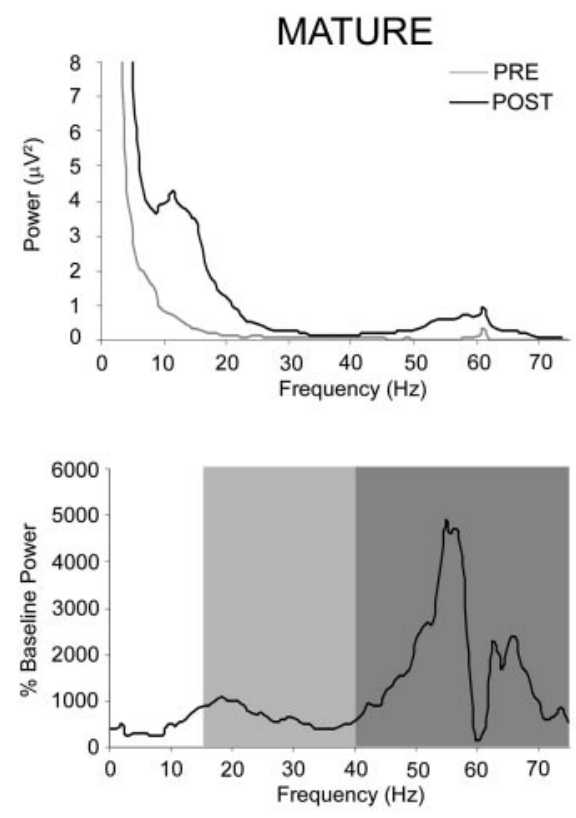

Figure 2. Comparison of olfactory bulb LFP activity in infant and mature rats presented with isoamyl acetate (150 ppm A, Mean power spectra of odor-evoked LFP oscillations in the olfactory bulb of 7-d-old and mature rats. Mean powe gray) bands in the mature rats as opposed to the theta/low $\beta$ activity seen in pups. Inset depicts an enlargement of $\gamma$ power for $\mathrm{P} 7$ rats plot on the same power scale as the mature data.

respiratory cycle showed much greater odor-evoked oscillatory activity in mature rats compared with P7 rats (Fig. 3). In P7 rats, odor stimulation induced a slight, but significant increase in the $\beta$-frequency range power (ANOVA: odor-evoked $\beta, F_{(7,63)}=2.1$; $p=0.05)$ with no significant increase in odor-evoked $\gamma$ activity (ANOVA: odor-evoked $\gamma, F_{(7,63)}=1.4$; NS) (Fig. $3 A$ ). In contrast, odor stimulation in the mature rats elicited a strong increase in $\beta$ band oscillations (ANOVA: odor-evoked $\beta, F_{(7,63)}=$ $5.3 ; p<0.05)$ as well as an increase in $\gamma$ frequencies not seen in the young animals (ANOVA: odor-evoked $\gamma, F_{(7,63)}=2.5 ; p<$ 0.05 ) (Fig. 3B). There was significantly greater odor-evoked $\beta$ and $\gamma$ power in the mature rats compared with the P7 rats (ANOVA: main effect of age, $F_{(1,36)}=9.4 ; p<0.05$ ).

Differences in odor-evoked LFP activity across age were also seen in the piriform cortex. The majority of odor-evoked activity seen in the piriform cortex of P7 rats consisted of $\beta$ band (15-40 $\mathrm{Hz}$ ) response and no detectable $\gamma$ band $(40-75 \mathrm{~Hz})$ activity (Fig. $4 A$ ). Time-frequency comparisons show oscillatory bursts contained much more odor-evoked $\beta$ band oscillatory activity and no significant detectable odor-evoked $\gamma$ band activity in both P7 (ANOVA: odor-evoked $\beta, F_{(7,49)}=4.9, p<0.05$; odor-evoked $\gamma$ : $F_{(7,49)}=2.1$, NS) (Fig. $4 C$ ) and mature rats (ANOVA: odorevoked $\beta, F_{(7,49)}=3.3, p<0.05$; odor-evoked $\left.\gamma: F_{(7,49)}=1.7, \mathrm{NS}\right)$ (Fig. $4 D$ ). The odor-evoked LFPs seen in the mature piriform cortex showed much greater oscillatory bursts per respiratory cycle than that seen in the P7 piriform cortex, and there was significantly greater odor-evoked $\beta$ band power in the mature rats compared with the P7 rats (ANOVA: main effect of age, $\left.F_{(1,28)}=8.9 ; p<0.05\right)$.

Comparisons of single-unit mitral/tufted cell spontaneous and odor-evoked activity revealed differences across age (Fig. 5). 

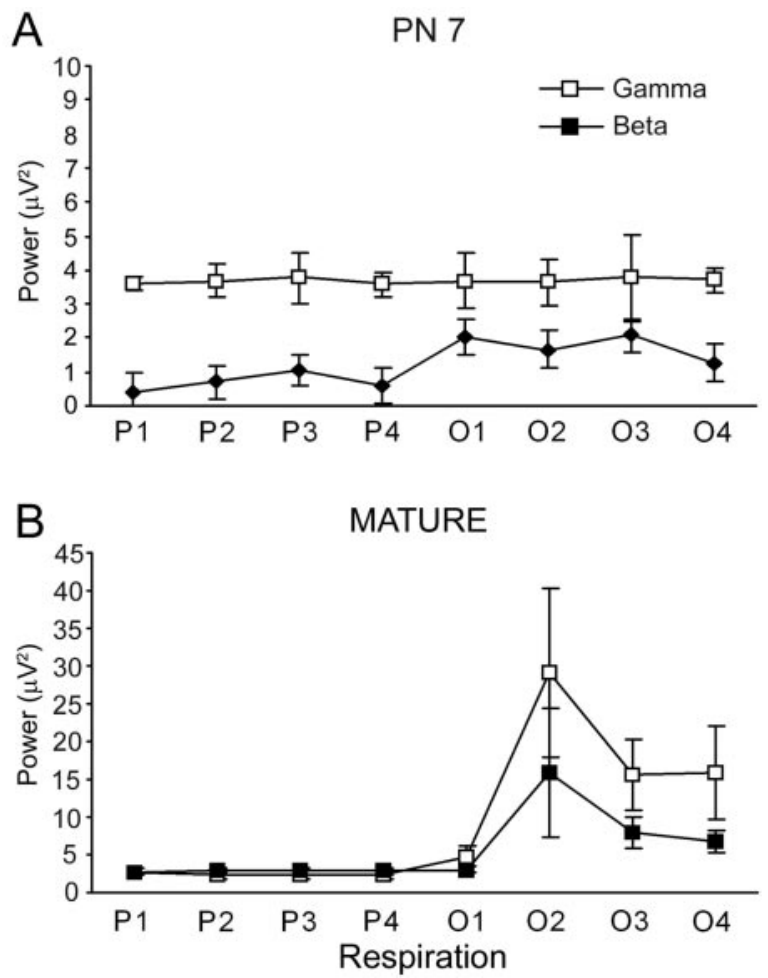

Figure 3. Comparison of the total mean power in the $\beta$-frequency $(15-40 \mathrm{~Hz})$ and $\gamma$-frequency $(40-75 \mathrm{~Hz})$ bands for each respiratory cycle during odor stimulation in P7 and mature rats. P1-P4 represents four respiratory cycles immediately before odor onset; $01-04$ represents four respiratory cycles during odor stimulation. Mean baseline (preodor) power spectra were calculated over four sniff cycles (P1-P4) with power spectra before and during (01-04) a 2 sodor stimulus expressed as a percentage of this baseline activity. Odor stimulation elicits stronger $\beta$ - and $\gamma$-frequency responses in the mature rats. Error bars denote $\pm \mathrm{SE}$.

Of the 77 total cells recorded, 15 cells in each age group responded to at least one ethyl ester and thus are included in the analysis here. Mean single-unit spontaneous activity was significantly greater in the mature rats than in P7 rats (mean spontane-
A

PN 7

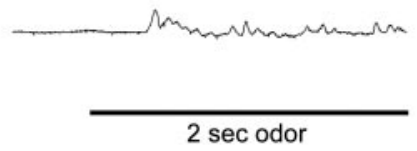

C

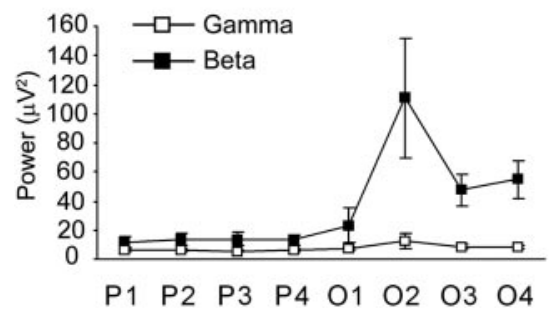

B

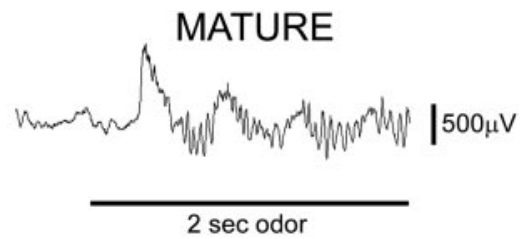

D

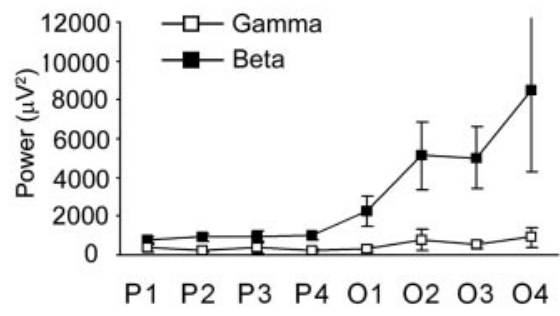

Figure 4. Examples of piriform cortex LFPs recorded before and during a 2 s presentation of isoamyl acetate (150 ppm dilution). $A$, Raw signal recorded from the piriform cortex of a 7-d-old rat. $B$, Raw signal recorded from the piriform cortex of a mature rat. The horizontal bar indicates odor presentation. C, D, Comparison of the total power in the $\beta$-frequency $(15-40 \mathrm{~Hz})$ and $\gamma$-frequency $(40-75 \mathrm{~Hz})$ bands for each respiratory cycle during odor stimulation in P7 and mature rats. Mean baseline (preodor) power spectra were calculated over four sniff cycles (P1-P4) with power spectra before and during (01-04) a 2 s odor stimulus expressed as a percentage of this baseline activity. The majority of odor-evoked piriform cortex activity in P7 and mature rats is in the $\beta$-frequency $(15-40 \mathrm{~Hz})$ ranges with minimal $\gamma$ band $(40-75 \mathrm{~Hz})$ activity.
A

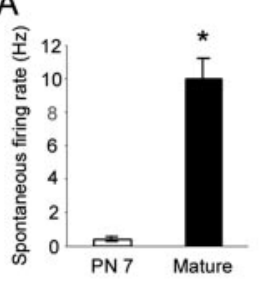

B

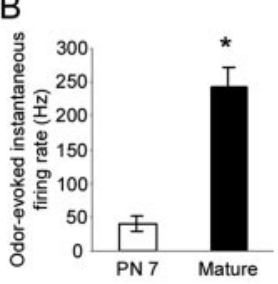

C

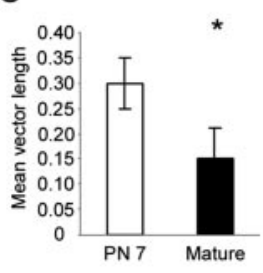

D

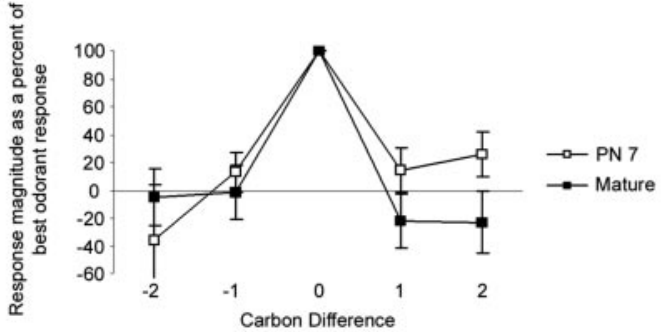

Figure 5. Mitral/tufted cell single unit activity in P7 and mature rats. $A$, Spontaneous activity was significantly elevated in adult rats compared with cells in P7 rats. B, Odor-evoked activity induced significantly higher firing rates in mature rats compared with P7 cells. C, Single-unit activity was significantly more tightly in phase with respiration in $\mathrm{P} 7$ rats than cells in mature rats. $D$, Mitral/tufted cell single-unit odorant receptive fields to a homologous series of ethyl esters (150 ppm) were not significantly different between cells in P7 and mature rats. Asterisks indicate significantly greater mean spontaneous activity in mature rats. Error bars represent SE.

ous activity: mature, $10.0 \pm 1.3 \mathrm{~Hz} ; \mathrm{P} 7,0.4 \pm 0.2 \mathrm{~Hz}$; unpaired $t$ test: $\left.t_{(28)}=-7.6, p<0.05\right)$ (Fig. $5 A$ ). Overall, odorants appeared to elicit more robust responses in the mature rats (mitral/tufted cell mean odor-evoked maximum instantaneous firing rate: mature, $242.3 \pm 30.8 \mathrm{~Hz}$; P7, $39.0 \pm 11.7 \mathrm{~Hz}$ ) (Fig. 5B). At P7, mitral/tufted activity was significantly more strongly in phase with respiration than mature mitral/tufted cell activity (mean vector length: P7, $0.30 \pm 0.05$; mature, $0.15 \pm 0.06$; MannWhitney $U$ test: $U=44.0 ; p<0.05$ ) (Fig. 5C). Thus, mitral/tufted cells in P7 rats displayed low levels of spontaneous activity, less robust odor-evoked activity, and activity more tightly in phase with respiration than cells in mature rats. Surprisingly, no significant differences were detected in the normalized mitral/tufted cell receptive fields (Fig. 5D) to a homologous series of equal concentration ethyl esters between age groups (ANOVA: main effect of odor, $F_{(4,109)}=18.6, p<0.05$; main effect of age, $F_{(4,109)}=1.2$, NS; odor by age, $F_{(4,109)}=$ $1.5, \mathrm{NS})$.

Despite the significant differences seen in odor-evoked activity in both the olfactory bulb and piriform cortex of P7 and mature rats, animals at all ages tested were able to discriminate ethyl esters using a heart rate-orienting response habituation/ cross-habituation paradigm (Fig. 6). Animals of all ages habituated to a single ethyl ester were able to discriminate structurally similar ethyl esters (ANOVA: main effect of age, $F_{(2,84)}=0.24$, NS; main effect of odor, $F_{(2,84)}=15.78, p<0.05$, no significant age $X$ odor interaction). Post hoc Fisher's analysis revealed a significant difference between the response magnitude to the habituated odor and to ethyl esters 1 


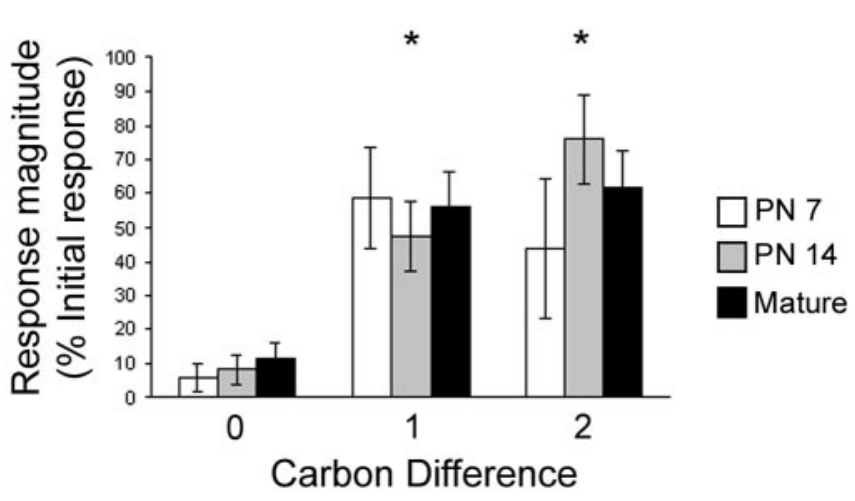

Figure 6. Discrimination of novel ethyl esters of different carbon chain length by P7, P14, and mature rats using a heart rate-orienting response habituation/cross-habituation paradigm. The amount of habituation ( 0 carbon difference) as well as the response magnitude to the cross-habituated odors is expressed as a percentage of the initial heart rate drop for that odor. Animals at all ages can discriminate ethyl esters differing by a single carbon at the $150 \mathrm{ppm}$ concentrations. Asterisks represent significant difference from habituated odor response. Error bars represent SE.

and 2 carbons longer for each age, $p<0.05$.

Because this study compared odor-evoked LFP activity in urethane-anesthetized animals to odor acuity in awake animals, a small number of awake olfactory bulb LFP recordings in P7 rats $(n=3)$ were also performed under temperature and odorstimulation conditions identical to the behavioral odor discrimination task. This was done to investigate any possible differences in LFP power in awake animals as well as differences related to body temperature. Although olfactory bulb LFPs from awake animals showed greater odor-evoked $\beta$ band oscillatory power than the anesthetized animals, no differences in odor-evoked $\gamma$ band activity were seen between anesthetized and awake $\mathrm{P} 7$ rats (ANOVA: condition $\times$ frequency, $F_{(1,22)}=10.5, p<0.05$; post hoc Fisher's test for $\beta$ power, $p<0.05 ; \gamma$, NS).

\section{Discussion}

Olfactory bulbs with reduced local interneurons may be expected to have poor odor quality coding according to either the spatial or temporal odor coding hypotheses. Together, these two often diametrically opposed hypotheses should predict that infant rats would be deficient in odor discrimination compared with mature animals, given the anatomical and functional immaturity of the infant rat olfactory system. This, however, was not the case. Based on data presented here, infant rats discriminate similar odors just as well as mature rats despite the restricted number of local interneurons and lack of odor-induced $\gamma$ oscillations compared with adults. These results raise questions as to the role of both local olfactory bulb interneurons and olfactory bulb LFP high-frequency oscillations in odor discrimination.

\section{Odor-evoked olfactory bulb local field-potential oscillations differ with age}

Odor-evoked LFP oscillations showed a strong age-dependent change in dominant frequencies between infant and mature rats. In $\mathrm{P7}$ rats, odor stimulation evoked a small increase in lower end $\beta$ band activity but no detectable increase in $\gamma$ oscillations, whereas in mature animals, odor stimulation evoked significantly larger $\beta$ and $\gamma$ responses (Fig. 2). The lack of odor-evoked $\gamma$ oscillations was observed in both urethane-anesthetized and awake pups. Similar results were seen in a previous study of spontaneous olfactory bulb LFPs in awake rats in that infants displayed a dominant frequency at $\sim 11 \mathrm{~Hz}$ on P6 with very few $\beta$ - and $\gamma$-frequency oscillations, and adults were found to have dominant frequencies well within the $\beta$ - and $\gamma$-frequency ranges (Almli et al., 1985).

Because olfactory bulb $\gamma$ oscillations are generated by mitral/ tufted-granule cell reciprocal interactions (Rall and Shepherd, 1968; Eeckman and Freeman, 1990; Neville and Haberly, 2003; Lagier et al., 2004), it is not surprising that infant rats would exhibit reduced odor-evoked oscillations considering that the number of granule cells is only $\sim 40 \%$ of the adult number (Rosselli-Austin and Altman, 1979; Bayer, 1983) and most do not reach adult-like morphology until the third postnatal week (Mair et al., 1982; Frazier-Cierpial and Brunjes, 1989). The immature granule cell population combined with largely unmyelinated mitral cell axons at this age (Schwob et al., 1984) could lead to a greatly reduced olfactory circuit that is incapable of generating the fast oscillations seen in the adult.

Differences in odor-evoked $\beta$ and $\gamma$ band activity were also seen in the piriform cortex (Fig. 4). Although there was no agedependent change in dominant frequency, odor-evoked changes between ages were seen. Most notably, adult rats showed stronger odor-evoked $\beta$ band power compared with the infants. This could be caused by slower conduction velocities of unmyelinated mitral cell axons and delayed maturation of cortical inhibition seen in infant rats (Schwob et al., 1984).

\section{Olfactory bulb mitral/tufted cell activity differs between ages, yet ORFs remain similar}

Single-unit mitral/tufted cell spontaneous and odor-evoked activity differed between ages (Fig. 5). Spontaneous activity was much greater in mature rats than in infants. This is consistent with other reports of mitral/tufted cell activity increasing with age (Math and Davrainville, 1980; Shafa et al., 1981; Mair and Gesteland, 1982; Wilson and Leon, 1986). Similarly, odorants appeared to elicit more robust responses in mature rats as measured by instantaneous firing rates. Mitral/tufted cell firing in relation to the respiratory cycle also differed between ages. At P7, mitral/tufted activity was significantly more in phase with respiration than mature mitral/tufted cell activity. These results suggest that the temporal response patterns of infant mitral/tufted cells more closely reflect olfactory receptor input than that of mature animals, perhaps because of reduced local circuit and cortical feedback activity in neonates.

Despite differences in spontaneous activity and odor-evoked instantaneous firing rates, no dramatic differences were observed between infant and mature single-unit mitral/tufted ORFs. Infant and adult rat mitral/tufted cell ORFs had a single best odorant with similar odorants evoking relatively smaller responses. This suggests that mitral/tufted cell responses to a particular odorant in relation to other related odorants are similar in infants and mature animals, again despite major differences in local circuit anatomy across ages.

\section{Discrimination of structurally similar esters is similar in infants and mature rats}

Despite the differences in olfactory bulb odor-evoked activity, no difference in discriminatory ability was observed, with animals at all ages capable of discriminating 1- and 2-carbon odorants at 150 ppm concentration. These results show infant rats' olfactory acuity to be equal to that of mature animals in a simple odor discrimination task.

In light of the differences between infant and mature rat olfactory bulb odor-evoked oscillatory activity, these results raise 
questions as to the role of granule cells and $\gamma$-frequency oscillations in olfactory odor coding.

\section{Odor coding and odor discrimination in neonates}

Our results support the view that changes in firing rates of spatially defined mitral/tufted cells may be sufficient for simple odor quality coding in the mammalian olfactory bulb. In this view the olfactory bulb acts as an odorant feature detector in which different features of a specific odorant activate specific sets of glomeruli and their corresponding mitral/tufted cells (Mori et al., 1999; Leon and Johnson, 2003). The piriform cortex then integrates these subsets of active cells (features) into perceptual wholes (Wilson and Stevenson, 2003), thus enabling the animal to discriminate a variety of odorants. This basic circuitry is relatively intact by P7 in the rat, as is simple odor discrimination of similar odorants. Large populations of local interneurons, and the LFP high-frequency oscillations dependent on those interneurons, are not present at $\mathrm{P} 7$, and yet there is no corresponding deficit in behavioral or mitral/tufted cell odorant discrimination. These results are consistent with findings in both Manduca moths (Christensen et al., 1998) and zebrafish (Friedrich et al., 2004) that odor-evoked synchronous output neuron oscillatory activity is not required for odor identity coding.

Odor-specific spatial patterns of glomerular activity are apparent at birth and do not change dramatically over the postnatal period, whereas spatial patterns of mitral cell activity are more diffuse early in development and then become spatially restricted with maturation (Astic and Saucier, 1981; Guthrie and Gall, 2003). The restriction of spatial patterns of mitral cell activity may reflect the increased numbers of inhibitory interneurons (Brunjes and Frazier, 1986) and/or the pruning of olfactory receptor axons (Zou et al., 2004) and mitral cell apical dendrites during the first and second postnatal weeks (Malun and Brunjes, 1996). Nonetheless, the present results suggest that spatial coding alone may be sufficient for discrimination between monomolecular compounds in the absence of background stimuli, intensity shifts, or complex odor plume dynamics. LFP high-frequency oscillations, on the hand, may indicate processing that is not required for simple discrimination.

Furthermore, work from a variety of behavioral paradigms has suggested that olfactory bulb LFP oscillations, rather than simply reflecting odor quality encoding, may reflect behavioral state, past experience, and odor expectancy (Freeman and Schneider, 1982; Gray and Skinner, 1988; Ravel et al., 2003). For example, in highly trained animals, $\gamma$-frequency oscillatory activity is often most pronounced before odor onset, at a time when the animal is preparing to sample the odor (Ravel et al., 2003). Similarly, Freeman has demonstrated that olfactory bulb spatial patterns of LFP oscillations (primarily reflecting mitral-granule cell interactions) are not odor-specific, but rather reflect past associations and memory (Freeman and Schneider, 1982).

These data suggest strong top-down influences on olfactory bulb LFP oscillations, which may not be expected to dramatically affect simple discrimination of novel odorants. Freeman suggests this cortical feedback may create expectancy-based templates of local interneuron (granule cell) activity, on which the receptor afferent activity is imposed (Freeman and Schneider, 1982). In situations such as the well learned tasks described above, this feedback could then facilitate odor recognition and discrimination. In other situations, such as the simple discrimination of novel odorants used here, such feedback (and the resulting highfrequency oscillations) may be less critical for optimal discrimination performance. Current work is focused on applying this model to investigate the effects of expectation and attention on odor discrimination in infant rats, with the hypothesis that neonates may be specifically impaired in expectancy-based effects on olfactory behavior.

The results observed here are reminiscent of the extensive literature on the effects of olfactory bulb lesions on simple odor discrimination by Slotnick and Bisulco (2003) and Slotnick et al. (2004). There is clear evidence that odorants evoke unique spatial patterns of glomerular layer activity in the olfactory bulb, but that large lesions within these patterns have no detectable effect on simple discrimination. These findings suggest that discrimination between monomolecular compounds, in the absence of background stimuli, intensity shifts or complex odor plume dynamics, is a remarkably simple task for the vertebrate olfactory system-a task easily solved by even a greatly reduced system (ontogenetically or experimentally).

The question remains then, given that the olfactory system can function apparently well despite restricted numbers of interneurons and associated LFP oscillations compared with the mature system, what is the role of these additional cells? It should be noted that in the mature mammalian olfactory bulb, the ratio of numbers of output neurons to juxtaglomerular neurons is 1:20 and output neurons to granule cells is 1:50 or higher (Shepherd, 1998). These ratios are extremely different than what is found in mammalian thalamus (Shepherd, 1998) and mammalian neocortex (Shepherd, 1998), which all express the reversed ratio of output neurons to local interneurons of $\sim 3: 1$. The mammalian olfactory bulb appears to be an extreme outlier in terms of local interneuron numbers, although it clearly functions relatively well on simple tasks with many fewer interneurons. The apparent robust redundancy and complexity of the mature olfactory system, therefore, may reflect the fact that odor-guided behavior normally requires much more sophisticated computation than is required for simple odor discrimination.

\section{References}

Adrian ED (1950) The electrical activity of the mammalian olfactory bulb. Electroencephalogr Clin Neurophysiol 2:377-388.

Almli CR, Henault MA, Velozo CA, Morgane PJ (1985) Ontogeny of electrical activity of main olfactory bulb in freely-moving normal and malnourished rats. Dev Brain Res 18:1-12.

Astic L, Saucier D (1981) Ontogenesis of the functional activity of rat olfactory bulb: autoradiographic study with the 2-deoxyglucose method. Brain Res 254:243-256.

Bayer SA (1983) 3H-thymidine-radiographic studies of neurogenesis in the rat olfactory bulb. Exp Brain Res 50:329-340.

Bressler SL, Freeman WJ (1980) Frequency analysis of olfactory system EEG in cat, rabbit, and rat. Electroencephalogr Clin Neurophysiol 50:19-24.

Brunjes PC, Frazier LL (1986) Maturation and plasticity in the olfactory system of vertebrates. Brain Res 396:1-45.

Chabaud P, Ravel N, Wilson DA, Mouly AM, Vigouroux M, Farget V, Gervais R (2000) Exposure to behaviourally relevant odour reveals differential characteristics in rat central olfactory pathways as studied through oscillatory activities. Chem Senses 25:561-573.

Christensen TA, Waldrop BR, Hildebrand JG (1998) Multitasking in the olfactory system: context-dependent responses to odors reveal dual GABA-regulated coding mechanisms in single olfactory projection neurons. J Neurosci 18:5999-6008.

Christensen TA, Lei H, Hildebrand JG (2003) Coordination of central odor representations through transient, non-oscillatory synchronization of glomerular output neurons. Proc Natl Acad Sci USA 100:11076-11081.

Eeckman FH, Freeman WJ (1990) Correlations between unit firing and EEG in the rat olfactory system. Brain Res 528:238-244.

Fletcher M, Wilson DA (2001) Ontogeny of odor discrimination: a method to assess novel odor discrimination in neonatal rats. Physiol Behav 74:589-593.

Fletcher ML, Wilson DA (2003) Olfactory bulb mitral-tufted cell plasticity: 
odorant-specific tuning reflects previous odorant exposure. J Neurosci 23:6946-6955.

Frazier-Cierpial LL, Brunjes PC (1989) Early postnatal differentiation of granule cell dendrites in the olfactory bulbs of normal and unilaterally odor-deprived rats. Dev Brain Res 47:129-136.

Freeman WJ (1978) Spatial properties of an EEG event in the olfactory bulb and cortex. Electroencephalogr Clin Neurophysiol 44:586-605.

Freeman WJ, Schneider W (1982) Changes in spatial patterns of rabbit olfactory EEG with conditioning to odors. Psychophysiology 19:44-56.

Friedrich RW, Habermann CJ, Laurent G (2004) Multiplexing using synchrony in the zebrafish olfactory bulb. Nat Neurosci 7:862-871.

Gray CM, Skinner JE (1988) Field potential response changes in the rabbit olfactory bulb accompany behavioral habituation during the repeated presentation of unreinforced odors. Exp Brain Res 73:189-197.

Guthrie KM, Gall C (2003) Anatomic mapping of neuronal odor responses in the developing rat olfactory bulb. J Comp Neurol 455:56-71.

Kay LM, Lancaster LR, Freeman WJ (1996) Reafference and attractors in the olfactory system during odor recognition. Int J Neural Syst 7:489-495.

Lagier S, Carleton A, Lledo PM (2004) Interplay between local GABAergic interneurons and relay neurons generates gamma oscillations in the rat olfactory bulb. J Neurosci 24:4382-4392.

Lam YW, Cohen LB, Wachowiak M, Zochowski MR (2000) Odors elicit three different oscillations in the turtle olfactory bulb. J Neurosci 20:749-762.

Laurent G (1999) A systems perspective on early olfactory coding. Science 286:723-728.

Laurent G (2002) Olfactory network dynamics and the coding of multidimensional signals. Nat Rev Neurosci 3:884-895.

Laurent G, Davidowitz H (1994) Encoding of olfactory information with oscillatory neural assemblies. Science 265:1872-1875.

Leon M, Johnson BA (2003) Olfactory coding in the mammalian olfactory bulb. Brain Res Brain Res Rev 42:23-32.

Mair RG, Gesteland RC (1982) Response properties of mitral cells in the olfactory bulb of the neonatal rat. Neuroscience 7:3117-3125.

Mair RG, Gellman RL, Gesteland RC (1982) Postnatal proliferation and maturation of olfactory bulb neurons in the rat. Neuroscience 7:3105-3116.

Malun D, Brunjes PC (1996) Development of olfactory glomeruli: temporal and spatial interactions between olfactory receptor axons and mitral cells in opossums and rats. J Comp Neurol 368:1-16.

Math F, Davrainville JL (1980) Electrophysiological study on the postnatal development of mitral cell activity in the rat olfactory bulb. Brain Res 190:243-247.
Mori K, Nagao H, Yoshihara Y (1999) The olfactory bulb: coding and processing of odor molecule information. Science 286:711-715.

Neville KR, Haberly LB (2003) Beta and gamma oscillations in the olfactory system of the urethane-anesthetized rat. J Neurophysiol 90:3921-3930.

Nusser Z, Kay LM, Laurent G, Homanics GE, Mody I (2001) Disruption of GABA(A) receptors on GABAergic interneurons leads to increased oscillatory power in the olfactory bulb network. J Neurophysiol 86:2823-2833.

Rall W, Shepherd GM (1968) Theoretical reconstruction of field potentials and dendrodendritic synaptic interactions in olfactory bulb. J Neurophysiol 31:884-915.

Ravel N, Chabaud P, Martin C, Gaveau V, Hugues E, Tallon-Baudry C, Bertrand O, Gervais R (2003) Olfactory learning modifies the expression of odour-induced oscillatory responses in the gamma $(60-90 \mathrm{~Hz})$ and beta $(15-40 \mathrm{~Hz})$ bands in the rat olfactory bulb. Eur J Neurosci 17:350-358.

Rosselli-Austin L, Altman J (1979) The postnatal development of the main olfactory bulb of the rat. J Dev Physiol 1:295-313.

Schwob JE, Haberly LB, Price JL (1984) The development of physiological responses of the piriform cortex in rats to stimulation of the lateral olfactory tract. J Comp Neurol 223:223-237.

Shafa F, Shineh SN, Bidanjiri` A (1981) Development of spontaneous activity in the olfactory bulb neurons of postnatal rat. Brain Res 223:409-412.

Shepherd GM, ed (1998) The synaptic organization of the brain., Fourth Edition. New York: Oxford UP.

Slotnick B, Bisulco S (2003) Detection and discrimination of carvone enantiomers in rats with olfactory bulb lesions. Neuroscience 121:451-457.

Slotnick B, Cockerham R, Pickett E (2004) Olfaction in olfactory bulbectomized rats. J Neurosci 24:9195-9200.

Uchida N, Takahashi YK, Tanifuji M, Mori K (2000) Odor maps in the mammalian olfactory bulb: domain organization and odorant structural features. Nat Neurosci 3:1035-1043.

Wilson DA (1998) Habituation of odor responses in the rat anterior piriform cortex. J Neurophysiol 79:1425-1440.

Wilson DA, Leon M (1986) Early appearance of inhibition in the neonatal rat olfactory bulb. Brain Res 391:289-292.

Wilson DA, Sullivan RM (1990) Olfactory associative conditioning in infant rats with brain stimulation as reward. I. Neurobehavioral consequences. Brain Res Dev Brain Res 53:215-221.

Wilson DA, Sullivan RM (1994) Neurobiology of associative learning in the neonate: early olfactory learning. Behav Neural Biol 61:1-18.

Wilson DA, Stevenson RJ (2003) The fundamental role of memory in olfactory perception. Trends Neurosci 26:243-247.

Zou DJ, Feinstein P, Rivers AL, Mathews GA, Kim A, Greer CA, Mombaerts P, Firestein S (2004) Postnatal refinement of peripheral olfactory projections. Science 304:1976-1979. 